\title{
Glioma de nervo óptico: achados neuro-oftalmológicos em paciente com neurofibromatose tipo I
}

\section{Optic nerve glioma: neuro-ophthalmologic findings in a patient with Neurofibromatosis type I}

Marco Túlio Chater Viegas', Valquise Yumi Murata ${ }^{2}$, Fabiana Richa Valim³ ${ }^{3}$ Thiago Pardo Pizarro

\begin{abstract}
ReSUmo
Este artigo relata o caso de uma criança do sexo feminino, de nove anos de idade, com história de proptose e baixa acuidade visual progressiva. Ao exame oftalmológico apresentava: acuidade visual com a melhor correção 20/20 em olho direito e percepção de luz à esquerda; biomicroscopia sem alterações nos dois olhos; com hipofunção dos músculos reto superior esquerdo e oblíquo inferior esquerdo; exotropia e hipotropia esquerda; fundoscopia à direita normal e edema de papila à esquerda. Á Tomografia Computadorizada, apresentou tumor hiperdenso, fusiforme, em trajeto do nervo óptico, sugestivo de gliose do nervo óptico.Ao exame físico constataram-se manchas "cafécom-leite" em região torácica e axilar e sardas axilares, preenchendo critérios para Neurofibromatose do tipo I (doença de Von Recklinghausen).
\end{abstract}

Descritores: Glioma do nervo óptico; Neurofibromatose /diagnóstico; Acuidade visual; Relato de casos [Tipo de publicação]

'Residente do terceiro ano de Oftalmologia da Faculdade de Medicina de Rio Preto - FAMERP- São José do Rio Preto- (SP) - Brasil; ${ }^{2}$ Residente do terceiro ano de Oftalmologia da Faculdade de Medicina de Rio Preto - FAMERP- São José do Rio Preto- (SP) - Brasil; ${ }^{3}$ Estagiária do primeiro ano de Oftalmologia da Faculdade de Medicina de Rio Preto - FAMERP - São José do Rio Preto - (SP) - Brasil; ${ }^{4}$ Estagiário do segundo ano de Oftalmologia da Faculdade de Medicina de Rio Preto - FAMERP- São José do Rio Preto- (SP) - Brasil. 


\section{INTRODUÇÃO}

A Neurofibromatose do tipo I (NF1), também denominada Neurofibromatose múltipla, Molluscum fibrosum ou doença cutânea de Von Recklinghausen, foi descrita por Frederick Daniel Von Recklinghausen em $1882^{(1)}$. Faz parte de um grupo de enfermidades denominadas de síndromes neurocutâneas ou facomatoses.

Este distúrbio pode tanto ocorrer esporadicamente, como de forma hereditária, sendo o último tipo mais comum. A transmissão é autossômica dominante com $50 \%$ de novas mutações com expressão altamente variável e penetrância quase completa ${ }^{(2.5)}$. Tais pacientes têm uma anormalidade no lócus 17q11.2. É um gene complexo, codificado por uma proteína chamada neurofibrina, que provocaria uma inativação do protooncogene p21ras, implicando em numerosos tumores no homem $^{(5)}$.

Estima-se que acometa 100.000 pessoas nos Estados Unidos, sendo considerado o distúrbio genético mais freqüentemente encontrado no atendimento primário à saúde ${ }^{(3)}$.

Quando a doença se expressa de maneira leve, pode passar despercebida, aspectos clínicos frustros devem ser buscados com cuidado ${ }^{(2)}$. É provável que todo pediatra tenha um caso em sua clínica, porém na maioria das vezes, não diagnosticada ${ }^{(0)}$.

A apresentação clínica é variável, podendo ser encontrados: neurofibromas plexiformes, neurinomas dos troncos nervosos, máculas café com leite, manchas lentiginosas, neurofibromas cutâneos, sardas axilares e em região inguinal, além de outras alterações melanodérmicas, displasias ósseas e alterações oculares como: neuroma plexiforme de pálpebra superior, nódulos irianos de Lisch, hamartomas, glaucoma congênito, neurofibromas de nervos conjuntivais, glioma do nervo óptico e do quiasma óptico (7).

Gliomas ópticos são achados freqüentes em pacientes portadores de neurofibromatose ${ }^{(8)}$. Entre estes gliomas, o astrocitoma é o mais encontrado ${ }^{(9)}$. A associação de neurofibromatose e gliomas das vias ópticas geralmente ocorre em crianças jovens, sendo incomum o crescimento do tumor e o aparecimento de sintomas após os 10 anos de idade ${ }^{(10)}$. Em alguns casos de neurofibromatose, os astrocitomas se apresentam em múltiplos focos ${ }^{(11)}$.

O glioma de nervo óptico faz diagnóstico diferencial com as patologias: pseudotumor inflamatório, miosite pós-infecciosa ${ }^{(12)}$, rabdomiossarcoma entre outros tumores orbitários.
Os gliomas múltiplos podem se localizar tanto em região supratentorial como infratentorial ${ }^{(11,13)}$. Bilaniuk $^{(14)}$ descreveu casos de neurofibromatose com gliomas múltiplos nas vias ópticas e tronco encefálico. Essas lesões múltiplas podem sugerir metástases nos exames de neuroimagem. Infiltrações difusas do encéfalo também são descritas. Têm sido denominadas de gliomas multicêntricos, sendo a histologia diferente nos vários focos ${ }^{(1415)}$.

\section{Relato de Caso}

T.R.G., 9 anos, negra, natural de Araçatuba (SP), compareceu ao serviço de Oftalmologia do Hospital de Base/Faculdade de Medicina de São José do Rio Preto (SP) no dia 19/01/2006, com quadro de exotropia, proptose e baixa acuidade visual à esquerda, com tempo de evolução de aproximadamente 6 meses.

Negou cirurgias oculares, trauma, uso de medicações ou colírios. Estava em acompanhamento por Ptiríase Rubra.

Apresentava-se ao exame oftalmológico, acuidade visual (AV) sem correção 20/25 no olho direito $(\mathrm{OD})$ e percepção de luz em olho esquerdo (OE). Com refração estática (+1,5 OD e plano OE) a AV alcançou 20/20 no OD e não apresentou melhora no OE.

Ao exame biomicroscópico apresentava em ambos os olhos: olhos calmos, córneas transparentes, que não coravam com fluoresceína, cristalinos transparentes, íris sem alterações, pupilas centradas e fotorreagentes.

A pressão intra-ocular era de $12 \mathrm{mmHg}$ em OD e $08 \mathrm{mmHg}$ em OE, apresentando digitopressão normal nos dois olhos.

As rotações estavam preservadas em olho direito e apresentava hipofunção do músculo reto superior à esquerda (-1) e hipofunção do músculo oblíquo inferior esquerdo (-1).

Ao exame de "cover-test" apresentava hipotropia OE e exotropia concomitante, sendo a exoftalmometria de 15 milímetros neste olho.

A aximetria medida pelo biômetro de Humphrey ( San Diego, CA, USA) era de 22,87 $\mathrm{mm}$ em olho direito e 22,13 mm em olho esquerdo.

Ao fundo de olho apresentava em olho direito: papila rósea, mácula sem alterações, escavação 0,1 , periferia sem alterações. Em olho esquerdo apresentava: edema de papila, mácula sem alterações, periferia sem alterações.

Foram solicitados os seguintes exames complementares: tomografia computadorizada (TC) de órbita, ultrassonografia OE, hemograma, coagulograma, velocidade de hemosedimentação (VHS), proteína C reativa 

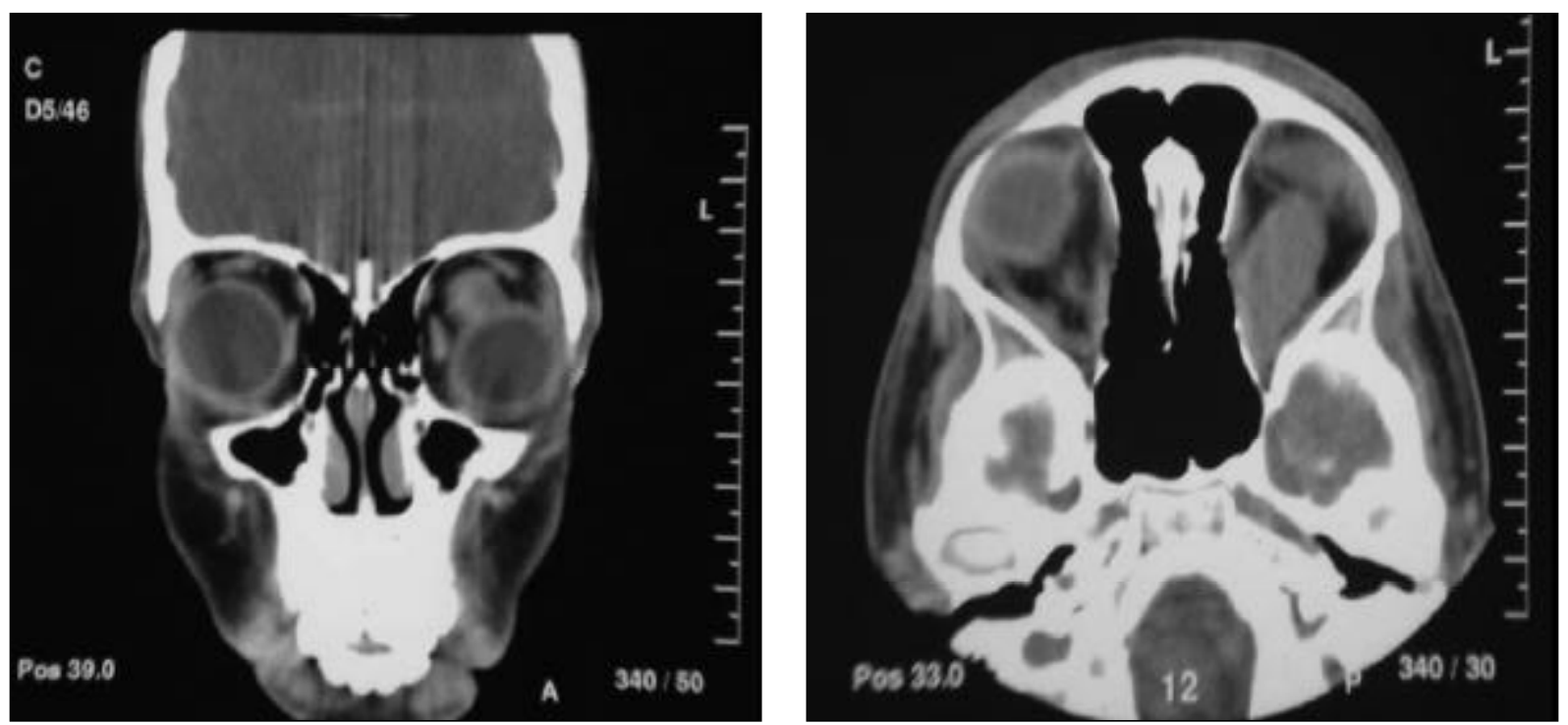

Figuras 1 e 2 - Aspecto de massa hiperdensa, fusiforme, em trajeto de nervo óptico

(PCR) e Mucoproteínas.

A paciente retornou no dia 31/01/2006 ao ambulatório, mantendo o quadro clínico inicial, trazendo os exames solicitados.

O hemograma, o coagulograma, mucoproteínas, VHS e a PCR apresentavam valores dentro dos limites da normalidade.

A TC orbitária evidenciou massa hiperdensa, fusiforme à esquerda, que acompanha trajeto do nervo óptico (Figuras 1 e 2). A musculatura extrínseca estava aparentemente sem infiltrações.

Ao exame físico, constatou-se "manchas cafécom-leite" em região cervical e torácica e múltiplas máculas (sardas) axilares.

A paciente foi então encaminhada ao serviço de Genética do Ambulatório do Hospital de Base - SJRP para avaliação e correlação dos achados clínico-radiológicos encontrados.

E posteriormente encaminhada ao serviço de neuroftalmologia do HC/ FMUSP em Ribeirão Preto, onde foi submetida a excisão cirúrgica, com craniotomia fronto-pterional com abertura de teto orbitário para ressecção da lesão. Evoluiu sem intercorrências, com amaurose, oftalmoparesia e ptose à esquerda.

\section{Discussão}

A paciente tem critérios clínicos para o diagnóstico de NF1 segundo o National Institutes of Health $(\mathrm{NIH})^{(16)}$; apresentou pelo menos três critérios cardinais: manchas café-com-leite, sardas (efélides) axilares e lesão compatível na TC com glioma do nervo óptico. Não foram encontrados, porém, neurofibromas, displasia óssea esfenóide, alterações palpebrais e nódulos de Lisch, que também são comumente encontrados na NF1 ${ }^{(17)}$.

O exame de TC pode identificar a lesão no nervo óptico, podendo ser descartados outros diagnósticos propostos.

Singhal et al. apresentou em seu trabalho 36 pacientes com glioma de nervo óptico ${ }^{(18)}$. O glioma esteve associado à NF1 em 50\% dos casos, com discreto predomínio em mulheres (52,9\%), com distúrbio visual como sintoma inicial de apresentação em $88 \%$ dos casos; diagnóstico realizado por TC em $60 \%$ dos casos e tratamento cirúrgico em $50 \%$ dos casos. Dados que foram compatíveis com este relato.

A idade até no máximo 10 anos para a apresentação dos sintomas e de diagnóstico da doença também estão em concordância com dados da literatura ${ }^{(10)}$.

Especificamente para o glioma, as possíveis condutas poderão ser: expectante, excisão cirúrgica com manutenção do bulbo ocular, radioterapia e quimioterapia ${ }^{(7)}$.

Muitos pacientes com glioma presente exclusivamente no trato óptico ainda podem preservar a AV razoável por alguns anos, sendo nestes casos considerado o tratamento conservador, com acompanhamento clínico e exames de neuroimagem a cada 6-12 meses ${ }^{(7,19)}$.

É necessário diferenciar os tipos de neurofibromatose ( tipo I e tipo II). A neurofibromatose 
do tipo II, também de herança autossômica dominante é menos comum que a neurofibromatose do tipo I e afeta um em cada 40.000 indivíduos ${ }^{(4)}$. A alteração genética está no cromossomo 22q12. As principais manifestações são neurinomas bilaterais no nervo acústico e tumores do sistema nervoso central. Os neuromas acústicos bilaterais se apresentam no final da adolescência e início da vida adulta, cursando com perda auditiva e alterações vestibulares. A maioria dos neuromas acústicos são schwanomas que se desenvolvem a partir no nervo vestibular. Em pacientes jovens o crescimento do tumor é invariavelmente rápido ${ }^{(4)}$. Existem alguns achados oftalmológicos tais como catarata (achado comum) e alguns incomuns como oftalmoplegia, hamartomas combinados do epitélio pigmentar da retina e membranas epirretinianas. A paciente em questão não apresentou os achados descritos acima, contribuindo para diferenciação entre as duas entidades nosológicas.

A excisão do tumor geralmente é realizada quando há perda da visão, proptose severa ou quando há extensão do tumor para o quiasma óptico. Além da tomografia computadorizada, foi solicitado ressonância nuclear magnética de crânio com injeção de contraste para melhor indicação e estadiamento da doença. Os achados foram: lesão expansiva na porção intraorbitária do nervo óptico à esquerda, caracterizada por hipersinal na ponderação T2 e hipointensa T1, com intenso realce após infusão do meio paramagnético. A lesão determina compressão sobre a margem superior do globo ocular à esquerda. Imagem de hipersinal na ponderação T2 localizada na região de núcleos da base à esquerda (globo pálido), medindo aproximadamente $1 \mathrm{~cm}$.

Optou-se pela excisão cirúrgica do tumor haja vista que existia baixa acuidade visual no olho acometido, sinais de edema de papila óptica e atrofia além de acometimento da região de núcleos da base (globo pálido).

Outro tipo de tratamento é a radioterapia, que parece retardar a progressão da doença, porém a radioterapia pode apresentar complicações como: alteração neuroendócrinas, deficits cognitivos e vasculopatia cerebral oclusiva, radionecrose cerebral, leucoencefalopatia e malignizações secundárias ${ }^{(7,20)}$.

A quimioterapia comprovadamente atrasa o uso de radioterapia, mas sem controle em longo prazo ${ }^{(7)}$.

A paciente apresentou alguns dados de bom prognóstico como nervo óptico, referentes à NF1, cujos tumores tendem a ser menos agressivos e com menor incidência de extensão para hipotálamo ${ }^{(21)}$.
Porém, por apresentar proptose e perda importante da acuidade visual, foi optado pela excisão cirúrgica do tumor. Ela deverá ser acompanhada continuadamente para descartar qualquer chance de recorrência do tumor, já que a taxa de recorrência após a excisão é de 0,5\%, valor que dobra em pacientes com $\mathrm{NF}^{(7)}$. Foi Orientado avaliação pós-cirúrgica multidisciplinar com oncopediatras, geneticistas e psicólogos.

\section{Abstract}

This article reports a case of a female child, nine-years old, with a history of proptosis and progressive decrease of visual acuity. At ophthalmologic exam presented: best corrected visual acuity 20/20 on the right eye and light perception on the left eye; biomicroscopic exam was normal, weakness of left superior rectus and left inferior oblique muscle, exotropia and hypotropia to the left. At indirect ophthalmoscopy exam, fundus was normal on the right eye and had left optic disc with edema. computerized tomography showed a hiperdense tumor, fusiform shape in the optic pathway, suggesting optic nerve glioma. At physical exam, it was verified "café-au-lait" spots in the thorax and armpits and axillary freckles in the region, filling criteria to neurofibromatosis type I (Von Recklinghausen's disease).

Keywords: Optic nerve glioma; Neurofibromatosis 1/diagnosis; Visual acuity; Case reports [Publication type]

\section{RefERÊNCIAS}

1. Von Recklinghausen F. Uber die multiplen fibrome der Haut und ihre Beziehung zu den multiplen Neuromen. Berlin: A. Hirschwald; 1882.

2. Caviness VS. Neurocutaneous syndromes and other developmental disorders of the central nervous system. In: Isselbacher KJ, Braunwald E, Wilson JD, Martin JB, Fauci AS, Kasper DL, Hauser SL. Harrison's principles of internal medicine. 13th ed. New York: McGraw-Hill; c1994. p 2333-44.

3. Cotran RS, Kumar V, Robbins SL, Shoen FJ. Robbins patologia estrutural e funcional. 5a ed. Rio de Janeiro: Guanabara Koogan; c1996.

4. Kanski JJ. Oftalmologia clínica: uma abordagem sistemática. 5 a ed. Rio de Janeiro: Elsevier; 2004. p. 655-702.

5. Wilkie AO, Amberger JS, McKusick VA. A gene map of congenital malformations. J Med Genet. 1994; 31(7): 507-17.

6. Goldberg Y, Dibbern K, Klein J, Riccardi VM, Graham JM Jr. Neurofibromatosis type 1 - an update and review for the primary pediatrician. Clin Pediatr (Phila). 1996; 35(11): 545-61.

7. Ellis BD. Optic pathway gliomas. In: Tasman W, Jaeger EA. Duane's Ophthalmology 2006 Edition (CD-ROM). Philadelphia: Lippincott Willians \& Wilkins; 2005. cap 42.

8. Stern J, DiGiacinto GV, Housepian EM. Neurofibromatosis and optic glioma: clinical and morphological correlations. Neurosurgery. 1979; 4(6):524-8. 
9. Mukai K, Kitamura K, Asano N, Ohshima T, Hondo H, Matsumoto K. [Multifocal gliomas in cerebral hemisphere associated with von Recklinghausen's disease: case report]. No Shinkei Geka. 1989; 17(2): 197-202. Japanese.

10. Bajenaru ML, Garbow JR, Perry A, Hernandez MR, Gutmann DH. Natural history of neurofibromatosis 1 - associated optic nerve glioma in mice. Ann Neurol. 2005; 57(1):119-27.

11. Nomura S, Susuki R, Sugiyama S, Nogami K, Ito H. Optic glioma with characteristics bilateral optic atrophy in a 3year-old girl. Pediatr Neurosurg. 1999; 31(4):213-8.

12. Rodrigues MPM, Dantas AM, Dantas MM, Dantas JM, Biancardi AL. Miosite ocular pós-infecciosa. Rev Bras Oftalmol. 2005; 64(5):348-50.

13. Jaskólski D, Zawirski M, Wisniewska G, Papierz W. A case of multicentric glioma of cerebellum and brain. Zentralbl Neurochir. 1988; 49(2):124-7.

14. Bilaniuk LT, Molloy PT, Zimmerman RA, Phillips PC, Vaughan SN, Liu GT, et al. Neurofibromatosis type 1: brain stem tumours. Neuroradiology. 1997; 39(9): 642-53.

15. Gupta SK, Nema HV, Bhatia PL, Sasibabu K, Kesharwani R. The radiological features of craniofacial neurofibromatosis. Clin Radiol. 1979; 30(5):553-7.
16. DeBella K, Szudek J, Friedman JM. Use of the national institutes of health criteria for diagnosis of neurofibromatosis 1 in children. Pediatrics. 2000; 105(3 Pt 1):608-14.

17. Curi CL, Herzog G, Sebastiá R. Manifestações órbitopalpebrais da neurofibromatose tipo 1: revisão de 16 casos. Arq Bras Oftalmol. 2004; 67(3): 429-32.

18. Singhal S, Birch JM, Kerr B, Lashford L, Evans DG. Neurofibromatosis type 1 and sporadic optic gliomas. Arch Dis Child. 2002; 87(1):65-70.

19. Aguirre-Balsalobre FE, Coloma-Gonzáles IT, Mengual-Verdú EN. Optic nerve glioma in a case of neurofibromatosis-1 in a child. Arch Soc Esp Oftalmol. 2006; 81(1):33-6. Id: Spa.

20. Grill J, Couanet D, Cappelli C, Habrand JL, Rodriguez D, Sainte-Rose C, Kalifa C. Radiation-induced cerebral vasculopathy in children with neurofibromatosis and optic pathway glioma. Ann Neurol. 1999; 45(3):393-6.

21. Tow SL, Chandela S, Miller NR, Avellino AM. Long-term outcome in children with gliomas of the anterior visual pathway. Pediatr Neurol. 2003; 28(4):262-70. 